\title{
Influence of combined oral contraceptives on polycystic ovarian morphology-related parameters in Korean women with polycystic ovary syndrome
}

\author{
Chan-Hong Park, MD', Sungwook Chun, MD, PhD² \\ ${ }^{1}$ Health Care Division, The Provincial Office of Jeollanamdo, Muan; ${ }^{2}$ Department of Obstetrics and Gynecology, Haeundae Paik Hospital, Inje University \\ College of Medicine, Busan, Korea
}

\section{Objective}

This study aimed to assess the effect of combined oral contraceptives (COCs) on polycystic ovarian morphology (PCOM) and serum anti-Müllerian hormone (AMH) levels in Korean women with polycystic ovary syndrome (PCOS).

\section{Methods}

This university hospital study enrolled 36 Korean women who were newly diagnosed with PCOS based on the Rotterdam criteria and were treated with COCs for at least 1 year. We retrospectively evaluated the ovarian volume and number of antral follicles using ultrasonography and assessed serum AMH levels at baseline and after 1 year of COC treatment.

Results

Significant decreases in ovarian volume and antral follicle count were observed after 1 year of COC treatment. Compared to baseline, serum AMH levels were significantly decreased after 1 year.

\section{Conclusion}

COC treatment significantly affects ultrasound-assessed PCOM and serum AMH levels of patients with PCOS.

Keywords: Anti-Müllerian hormone; Combined oral contraceptives; Polycystic ovarian morphology; Polycystic ovary syndrome

\section{Introduction}

Polycystic ovary syndrome (PCOS) is a common endocrine disorder affecting $8-13 \%$ women of reproductive age $[1,2]$. Common clinical characteristics include chronic anovulation, hyperandrogenism (hyperandrogenemia or clinical hyperandrogenism), and polycystic ovaries on ultrasonography. Infertility caused by chronic ovulatory dysfunction, abnormal gonadotropin secretion [3], and metabolic disturbances, such as central obesity, dyslipidemia, insulin resistance, and hyperinsulinemia, can be simultaneously encountered in PCOS [4].

The Rotterdam criteria have been widely used for the diagnosis of PCOS [5]. PCOS is diagnosed in women with at least 2 of the following: oligo-anovulation, clinical and/or biochemical hyperandrogenism, and polycystic ovarian morphology (PCOM) on ultrasound ( $\geq 12$ follicles measuring 2-9 mm in diameter in at least 1 ovary, and/or increased ovarian vol- ume to $>10 \mathrm{~cm}^{3}$ ) after the exclusion of androgen excesses.

Anti-Müllerian hormone (AMH), produced in the granulosa cells of small antral and preantral follicles, is a type of transforming growth factor $\beta$ [6]. AMH plays a critical role in

Received: 2019.04.12. Revised: 2019.08.24. Accepted: 2019.09.26. Corresponding author: Sungwook Chun, MD, PhD

Department of Obstetrics and Gynecology, Inje University Haeundae Paik Hospital, 875 Haeun-daero, Haeundae-gu, Busan 48108, Korea

E-mail: wooki1974@empal.com

https://orcid.org/0000-0002-9948-0360

Articles published in Obstet Gynecol Sci are open-access, distributed under the terms of the Creative Commons Attribution Non-Commercial License (http://creativecommons. org/licenses/by-nc/3.0/) which permits unrestricted non-commercial use, distribution, and reproduction in any medium, provided the original work is properly cited.

Copyright $\odot 2020$ Korean Society of Obstetrics and Gynecology 


\section{Obstetrics \& Gynecology Science}

Chan-Hong Park, et al. PCOM change during COC use in Korea

chronic anovulation by interrupting the initiation of primordial follicular growth [7] and promoting the arrest of follicular development [8]. AMH was recently recognized as a useful clinical marker for evaluating PCOS [9] and is considered promising for the accurate detection of PCOM [10].

Combined oral contraceptives (COCs), along with lifestyle modifications, are recommended for the primary management of PCOS to treat clinical symptoms such as irregular menstrual cycles, hirsutism, weight gain, and associated lipid, glucose, and testosterone disturbances [10]. However, in previous studies, treatment effects varied in association with the heterogeneous composition of COCs, treatment duration, symptom severity/phenotype, and accessibility of the treatment regimen [11-14]. Reports on the effects of COCs on PCOM and AMH among Korean women are lacking.

Thus, we aimed to examine the effect of COC treatment on PCOM and serum AMH levels in Korean patients with PCOS.

\section{Materials and methods}

\section{Patient population and study design}

This retrospective study included Korean women aged 18-35 years who were diagnosed with PCOS during their first visit to Inje University Haeundae Paik Hospital between June 2010 and December 2014; these women were followed up with transvaginal (TV-US) or transrectal ultrasound (TR-US) and serum AMH levels after 1 year of COC treatment. PCOS was diagnosed according to the Rotterdam criteria [5]. PCOS is diagnosed in women with at least 2 of the following: oligo-anovulation, clinical and/or biochemical hyperandrogenism, and PCOM on ultrasonography; other pathologic conditions such as non-classical congenital adrenal hyperplasia, androgen-producing tumors, hyperprolactinemia, Cushing's syndrome, and thyroid dysfunction were ruled out. Oligomenorrhea is defined as an interval of $>35$ days between menstrual cycles, while amenorrhea is defined as an interval of $>90$ days between menstrual cycles. In our trial, clinical hyperandrogenism was defined as the presence of hirsutism based on a Ferriman-Gallwey score of $>6$, modified for Korean women by Kim et al. [15]; biochemical hyperandrogenism was defined as a serum testosterone level exceeding 95\% confidence limits, as reported by Chae at al. [16] (total testosterone level $>0.68 \mathrm{ng} / \mathrm{mL}$ and/or free testosterone level $>1.72 \mathrm{pg} / \mathrm{mL}$ ). PCOM was defined as follows: $\geq 12$ follicles measuring 2-9 $\mathrm{mm}$ in diameter in each ovary and/ or an ovarian volume of $>10 \mathrm{~mL}$ on TV-US or TR-US. Exclusion criteria were as follows: prior ovarian surgery, suspected ovarian malignancy, or known contraindications to estrogen therapy and use of oral contraceptives, ovulation-inducing drugs, glucocorticoids, or anti-androgenic agents, which can affect the hypophysis-pituitary-ovarian axis in the 6 months preceding the diagnosis. Finally, 36 patients were included in our study. All patients were given a 24-day regimen of COCs containing ethinylestradiol (EE) $20 \mu \mathrm{g}$ and drospirenone (Yaz $\left.{ }^{\circledR}\right)$ $3 \mathrm{mg}$.

\section{Assessment of ovarian volume and antral follicle count}

All subjects were followed up with TV-US or TR-US to assess ovarian volume and antral follicle count. The TV-US or TRUS examination was performed using a Voluson S7 device (General Electric Systems, Seongnam, Korea) with a 7-MHz transvaginal probe. The baseline ultrasound was conducted at the early follicular phase, and the ultrasound after 1 year of treatment was performed during the pill-free interval (7-21 days) of the COC treatment. All the ultrasound examinations were conducted by the same reproductive endocrinologist in accordance with the international ultrasound definitions of PCOM [17]. The number of antral follicles measuring 2-9 $\mathrm{mm}$ in diameter was calculated using a continuous scanning method for each ovary from the inner to outer margins of a longitudinal cross-section. Ovarian volume was calculated according to a simplified formula for a prolate ellipsoid (0.5xlength $\times$ widthxthickness) [17]. Total ovarian volume (TOV) was calculated as the sum of the volume of the right ovary (ROV) and left ovary (LOV), and total antral follicle count (TFC) was calculated as the sum of the follicle count of the right ovary (RFC) and left ovary (LFC).

\section{Measurement of serum anti-Müllerian hormone levels}

Blood samples were collected in tubes without anticoagulants on the day of ultrasound examination, and the biochemical parameters of the serum were measured. Serum $\mathrm{AMH}$ levels were determined using an AMH Gen II assay (Beckman Coulter Inc., La Brea, CA, USA).

\section{Statistical analysis}

Data are expressed as mean \pm standard deviation and ana- 


\title{
Obstetrics \& Gynecology Science
}

\author{
Vol. 63 , No. 1, 2020
}

lyzed using SPSS version 25.0 (SPSS, Chicago, IL, USA). A paired samples $t$-test was used to compare parameters related to ovarian follicle count (TFC, RFC, and LFC), ovarian volume (TOV, ROV, and LOV), and AMH levels at baseline and after 1 year of treatment. Statistical significance was set at $P<0.05$.

\section{Results}

Baseline clinical characteristics are shown in Table 1. Table 2 shows that the baseline ultrasound parameters related to PCOM were significantly decreased after 1 year of COC treatment. There was a significant decrease in TFC from baseline (73.83 \pm 43.14$)$ to 1 year $(44.11 \pm 28.09)(40.3 \%$ decrease, $P<0.001)$. In addition, TOV decreased significantly after 1 year of treatment (from $24.57 \pm 8.39$ to $14.13 \pm 5.92 \mathrm{~cm}^{3}$; $P<0.001)$. Serum AMH levels were decreased significantly from $11.09 \pm 4.6 \mathrm{ng} / \mathrm{mL}$ at baseline to $6.45 \pm 3.29 \mathrm{ng} / \mathrm{mL}$ after 1 year of COC treatment $(P<0.001)$.

\section{Discussion}

PCOM is 1 of 3 items needed for diagnosis of PCOS. The

Table 1. Baseline patient characteristics $(n=36)$

\begin{tabular}{lc}
\hline Characteristics & Participants \\
\hline Age $(\mathrm{yr})$ & $25.03 \pm 5.35$ \\
Height $(\mathrm{cm})$ & $161.62 \pm 6.00$ \\
Body weight $(\mathrm{kg})$ & $56.18 \pm 13.24$ \\
Body mass index & $21.36 \pm 4.04$ \\
Waist to hip ratio & $0.77 \pm 0.06$ \\
TFC & $73.83 \pm 43.14$ \\
RFC & $39.31 \pm 21.54$ \\
LFC & $34.53 \pm 22.35$ \\
TOV $\left(\mathrm{cm}^{3}\right)$ & $24.57 \pm 8.39$ \\
ROV $\left(\mathrm{cm}^{3}\right)$ & $13.65 \pm 5.84$ \\
LOV $\left(\mathrm{cm}^{3}\right)$ & $10.91 \pm 4.11$ \\
AMH level $(\mathrm{ng} / \mathrm{mL})$ & $11.09 \pm 4.61$ \\
\hline
\end{tabular}

Data was expressed as mean \pm standard deviation.

TFC, total follicle count; RFC, right ovarian follicle count; LFC, left ovarian follicle count; TOV, total ovarian volume; ROV, right ovarian volume; LOV, left ovarian volume; $\mathrm{AMH}$, anti-Müllerian hormone.
2 cardinal features of PCOM include increases in ovarian volume and AFC levels. The AMH level is correlated with the number of follicles that are $2-5 \mathrm{~mm}$ in diameter [18]; serum AMH level is useful for assessing PCOM in patients with PCOS. COCs are recommended as primary treatment for chronic anovulation and hyperandrogenism in PCOS [10]. However, adequate research on the effects of COCs on PCOM in women with PCOS is lacking, and to the best of our knowledge, no such research has been performed among Korean women. We found a significant decrease in baseline TFC, TOV, and AMH values after 1 year of COC treatment in Korean patients with PCOS.

Several studies have investigated whether COCs can modulate PCOM in patients with PCOS [13,19-25]. According to Mes-Krowinkel et al. [19], the mean ovarian volume, mean ovarian follicle count, and AMH level remarkably decreased in patients using COCs than in patients who had never used COCs after correction for confounding variables such as body mass index, age, waist-to-hip ratio, previous pregnancy, infertility treatment, and ethnicity. Another study [20] reported that ovarian volume (ROV, LOV, and TOV), ovarian follicle count (RFC, LFC, and TFC), and AMH level were significantly decreased after 3 months of $\mathrm{COC}$ treatment. The authors suggested that the AMH level decreased due to COC-induced suppression of ovarian function. They used cyproterone acetate (CPA) 2 mg/EE (Diane 35) $35 \mu \mathrm{g}$; however, per current recommendations [10], this should not be first-line treatment because of adverse effects such as venous thromboembolism. Moreover, the AMH assay kits used

Table 2. Serial changes in ultrasound parameters of ovarian size and serum anti-Müllerian hormone levels $(n=36)$

\begin{tabular}{lccc}
\hline Parameters & Basal & Post 1 year & $\boldsymbol{P}$-value \\
\hline TFC & $73.83 \pm 43.14$ & $44.11 \pm 28.09$ & $<0.001$ \\
RFC & $39.31 \pm 21.54$ & $23.19 \pm 14.92$ & $<0.001$ \\
LFC & $34.53 \pm 22.35$ & $20.91 \pm 13.78$ & $<0.001$ \\
TOV $\left(\mathrm{cm}^{3}\right)$ & $24.57 \pm 8.39$ & $14.13 \pm 5.92$ & $<0.001$ \\
ROV $\left(\mathrm{cm}^{3}\right)$ & $13.65 \pm 5.84$ & $7.53 \pm 3.43$ & $<0.001$ \\
LOV $\left(\mathrm{cm}^{3}\right)$ & $10.91 \pm 4.11$ & $6.59 \pm 3.07$ & $<0.001$ \\
AMH level $(\mathrm{ng} / \mathrm{mL})$ & $11.09 \pm 4.61$ & $6.45 \pm 3.29$ & $<0.001$ \\
\hline
\end{tabular}

Data was expressed as mean \pm standard deviation.

TFC, total follicle count; RFC, right ovarian follicle count; LFC, left ovarian follicle count; TOV, total ovarian volume; $\mathrm{AMH}$, anti-Müllerian hormone; LOV, left ovarian volume; ROV, right ovarian volume. ${ }^{a)} P$, paired samples $t$-test. 


\section{Obstetrics \& Gynecology Science}

Chan-Hong Park, et al. PCOM change during COC use in Korea

differed from those in our study. Plouvier et al. [21] reported a significant decrease in follicle numbers per ovary and $\mathrm{AMH}$ levels after a 3-month trial of COCs. Fábregues et al. [22] reported significant TFC reductions after 6 months of COC use, with a significant positive correlation between TFC and AMH level (rho=0.92 and 0.88; $P=0.01$ and 0.004 , respectively). They used desogestrel $2 \mathrm{mg} / \mathrm{EE} 20 \mu \mathrm{g}$ as COC therapy in their research. Another study [23] reported that AMH, ROV, and LOV were significantly decreased after 6 months of COC use and that there was a significant correlation between LOV and $\mathrm{AMH}$ level at baseline and those after 6 months of treatment (rho=0.336 and 0310; $P=0.018$ and 0.034 , respectively). All patients in this study were adolescents with PCOS aged 13-17.5 years taking COCs containing drospirenone $3 \mathrm{mg} / \mathrm{EE} 30 \mu \mathrm{g}$.

Our results were not comparable to those of 2 previous studies. Panidis et al. [24] reported changes in AMH levels in 2 other COC treatment groups (CPA 2 mg/EE $35 \mu \mathrm{g}$ and drospirenone $3 \mathrm{mg} / \mathrm{EE} 2 \mu \mathrm{g}$ ) at 3 time points (baseline, 3 months, and 6 months). AMH level was significantly decreased in the CPA $2 \mathrm{mg} / \mathrm{EE} 35 \mu \mathrm{g}$ group, but not in the drospirenone $3 \mathrm{mg} / \mathrm{EE} 2 \mu \mathrm{g}$ group. However, the definition of PCOS in that study was based on the 1990 National Institutes of Health criteria for PCOS, and we used a different AMH assay kit in our study. Somunkiran et al. [25] reported that TFC and TOV, but not AMH level, were decreased after 6 months of therapy. However, the COC formula (CPA 2 mg/ $\mathrm{EE} 35 \mu \mathrm{g}$ ) and $\mathrm{AMH}$ assay kit differed from those in our study.

PCOS exhibits an abnormal gonadotropin secretion pattern. The increasing frequency of pulsatile gonadotropin-releasing hormone secretion at the level of the hypothalamus accelerates transcription of the $\beta$-subunit of luteinizing hormone (LH) more than that of the same subunit of follicle-stimulating hormone (FSH) [26] and increases the amplitude and pulse frequency of LH secretion in the pituitary [27]. As a result, an increase in the LH/FSH ratio and serum LH level is observed in patients with PCOS. Normal ovulation requires the coordinated and synchronized interaction of $\mathrm{LH}, \mathrm{FSH}$, insulin-like growth factor, and $\mathrm{AMH}$. However, in PCOS, inappropriate gonadotropin secretion inhibits the selection of dominant follicles and causes follicular arrest at an early antral stage [2]. Consequently, PCOM and AMH levels increase. Chun [28] proposed that TOV and TFC are significantly correlated with the LH/FSH ratio and serum LH level; in addition, changes in PCOM findings could reflect post-treatment changes in gonadotropin release. Excess AMH production might be associated with an increased antral follicle count caused by intra-ovarian androgen overabundance [24]. Drospirenonecontaining COCs may decrease serum androgen and gonadotropin levels; these combined effects of COCs could contribute to changes in PCOM and AMH levels in patients with PCOS.

Our study showed that COC treatment can improve PCOM (ovarian volume and follicle counts) and AMH levels, although the mean values in our participants still satisfied the diagnostic criteria of PCOM after 1 year of treatment. This result is in agreement with that reported by Mes-Krowinkel et al. [19] and may indicate that PCOM is relatively stable during $C O C$ treatment and could be a reliable marker for PCOS.

Insulin resistance contributes to the pathology of PCOS and is a cause of abnormal steroidogenesis within the ovaries $[2,27]$. Several studies have assessed whether AMH levels affect insulin resistance. According to Chun [29], patients with PCOS and a high AMH level $(\geq 10.0 \mathrm{ng} / \mathrm{mL})$ had a significantly higher 1-hour postprandial glucose (PPG1) level than those with an $\mathrm{AMH}$ level of $<10 \mathrm{ng} / \mathrm{mL}$. PPG1 is considered a better predictor of the risk of developing type 2 diabetes mellitus than 2-hour postprandial glucose level [30]. Nardo et al. [31] reported that AMH levels increased with insulin levels based on the Homeostatic Model Assessment of Insulin Resistance (HOMA-IR). Skałba et al. [32] reported a significant correlation of serum $\mathrm{AMH}$ and total or low-density lipoprotein cholesterol levels with HOMA-IR and a significant negative correlation between high-density lipoprotein cholesterol and $\mathrm{AMH}$ levels. According to a previous study [33], total cholesterol level showed a positive correlation with the LH/ FSH ratio. However, other researchers reported no correlation of $\mathrm{AMH}$ and PCOM with insulin-linked metabolic parameters [34-36].

In our research, PCOM was defined according to the Rotterdam criteria [5]. Based on newly revised recommendations in 2018 [10], however, the diagnostic criteria for PCOS have changed to some extent, especially with regard to ultrasound and PCOM. The diagnostic threshold for PCOM in either ovary has been revised as follows: $\geq 20$ follicles per ovary and/ or ovarian volume of $\geq 10 \mathrm{~mL}$ in either ovary using an endovaginal ultrasound transducer with a frequency bandwidth that includes $8 \mathrm{MHz}$ after ensuring that the corpus luteum, 


\title{
Obstetrics \& Gynecology Science
}

\author{
Vol. 63, No. 1, 2020
}

cysts, or dominant follicles are absent. Based on the newly revised recommendations [37] for determining the number of follicles per ovary, the authors analyzed 11 studies including 2,961 adult patients, and the data suggested optimal sensitivity and specificity at $>19$ follicles per ovary. They noted that the limitations of their work included the heterogeneous population (patients with and without PCOS) and use of $95 \%$ cutoff values to define abnormality. Further studies based on the revised 2018 recommendations are warranted.

The limitations of our study include its retrospective design and small sample size. Most patients in our study visited our hospital for oligo-anovulation or infertility rather than symptoms associated with hyperandrogenism. Therefore, the number of PCOS patients with clinical and/or biochemical hyperandrogenism might be lower in our study group than in the general PCOS patient population.

Our study was designed to compare PCOM and $\mathrm{AMH}$ levels at baseline and after 1 year of COC treatment in contrast with other studies that had a follow-up duration of $\leq 6$ months.

In conclusion, our study showed that COC treatment significantly influences both ultrasound and serum parameters of PCOM and PCOS. To the best of our knowledge, this is the first study to investigate the effects of COCs on PCOMrelated ultrasound and serum parameters among Korean women. Undoubtedly, the purpose of COC treatment in women with PCOS is managing hyperandrogenism and/or irregular menstrual cycles [37] and not to cure PCOS. In this respect, the clinical significance of the effect of COCs on PCOM in women with PCOS remains unclear. Further prospective large-scale trials will help confirm these preliminary findings.

\section{Conflict of interest}

No potential conflict of interest relevant to this article was reported.

\section{Ethical approval}

The study received approval from the Inje University Haeundae Paik Hospital Institutional Review Board (IRB No. 1297922014-035)

\section{References}

1. Teede H, Deeks A, Moran L. Polycystic ovary syndrome: a complex condition with psychological, reproductive and metabolic manifestations that impacts on health across the lifespan. BMC Med 2010;8:41.

2. Azziz R, Carmina E, Chen Z, Dunaif A, Laven JS, Legro $R S$, et al. Polycystic ovary syndrome. Nat Rev Dis Primers 2016;2:16057.

3. Arroyo A, Laughlin GA, Morales AJ, Yen SS. Inappropriate gonadotropin secretion in polycystic ovary syndrome: influence of adiposity. J Clin Endocrinol Metab 1997:82:3728-33.

4. Carmina E, Lobo RA. Polycystic ovary syndrome (PCOS): arguably the most common endocrinopathy is associated with significant morbidity in women. J Clin Endocrinol Metab 1999;84:1897-9.

5. The Rotterdam ESHRE/ASRM-Sponsored PCOS Consensus Workshop Group. Revised 2003 consensus on diagnostic criteria and long-term health risks related to polycystic ovary syndrome. Fertil Steril 2004;81:19-25.

6. Weenen C, Laven JS, Von Bergh AR, Cranfield M, Groome NP, Visser JA, et al. Anti-Müllerian hormone expression pattern in the human ovary: potential implications for initial and cyclic follicle recruitment. Mol Hum Reprod 2004;10:77-83.

7. Durlinger AL, Gruijters MJ, Kramer P, Karels B, Ingraham HA, Nachtigal MW, et al. Anti-Müllerian hormone inhibits initiation of primordial follicle growth in the mouse ovary. Endocrinology 2002;143:1076-84.

8. Durlinger AL, Gruijters MJ, Kramer P, Karels B, Kumar TR, Matzuk MM, et al. Anti-Müllerian hormone attenuates the effects of FSH on follicle development in the mouse ovary. Endocrinology 2001;142:4891-9.

9. Lee JR, Kim SH. Anti-Mullerian hormone and female reproduction. Korean J Obstet Gynecol 2009;52:285-300.

10. Teede HJ, Misso ML, Costello MF, Dokras A, Laven J, Moran $L$, et al. Recommendations from the international evidence-based guideline for the assessment and management of polycystic ovary syndrome. Hum Reprod 2018;33:1602-18.

11. Bhattacharya SM, Jha A, DasMukhopadhyay L. Comparison of two contraceptive pills containing drospirenone and $20 \mu \mathrm{g}$ or $30 \mu \mathrm{g}$ ethinyl estradiol for polycystic ovary syndrome. Int J Gynaecol Obstet 2016;132:210-3. 


\section{Obstetrics \& Gynecology Science}

Chan-Hong Park, et al. PCOM change during COC use in Korea

12. Chung JP, Yiu AK, Chung TK, Chan SS. A randomized crossover study of medroxyprogesterone acetate and Diane-35 in adolescent girls with polycystic ovarian syndrome. J Pediatr Adolesc Gynecol 2014;27:166-71.

13. Kriplani A, Periyasamy AJ, Agarwal N, Kulshrestha $V$, Kumar A, Ammini AC. Effect of oral contraceptive containing ethinyl estradiol combined with drospirenone vs. desogestrel on clinical and biochemical parameters in patients with polycystic ovary syndrome. Contraception 2010;82:139-46.

14. Ozdemir S, Görkemli H, Gezginç K, Ozdemir M, Kiyici A. Clinical and metabolic effects of medroxyprogesterone acetate and ethinyl estradiol plus drospirenone in women with polycystic ovary syndrome. Int J Gynaecol Obstet 2008;103:44-9.

15. Kim JJ, Chae SJ, Choi YM, Hwang SS, Hwang KR, Kim $\mathrm{SM}$, et al. Assessment of hirsutism among Korean women: results of a randomly selected sample of women seeking pre-employment physical check-up. Hum Reprod 2011;26:214-20.

16. Chae SJ, Kim JJ, Choi YM, Hwang KR, Jee BC, Ku SY, et al. Clinical and biochemical characteristics of polycystic ovary syndrome in Korean women. Hum Reprod 2008;23:1924-31.

17. Balen AH, Laven JS, Tan SL, Dewailly D. Ultrasound assessment of the polycystic ovary: international consensus definitions. Hum Reprod Update 2003;9:505-14.

18. Pigny P, Merlen E, Robert Y, Cortet-Rudelli C, Decanter $C$, Jonard $\mathrm{S}$, et al. Elevated serum level of anti-mullerian hormone in patients with polycystic ovary syndrome: relationship to the ovarian follicle excess and to the follicular arrest. J Clin Endocrinol Metab 2003;88:5957-62.

19. Mes-Krowinkel MG, Louwers YV, Mulders AG, de Jong $\mathrm{FH}$, Fauser BC, Laven JS. Influence of oral contraceptives on anthropomorphometric, endocrine, and metabolic profiles of anovulatory polycystic ovary syndrome patients. Fertil Steril 2014;101:1757-1965.e1.

20. Ozay AC, Emekci Ozay O, Okyay RE, Cagliyan E, Kume T, Gulekli B. Different effects of myoinositol plus folic acid versus combined oral treatment on androgen levels in PCOS women. Int J Endocrinol 2016;2016:3206872.

21. Plouvier P, Peigné $M$, Gronier $H$, Robin $G$, CatteauJonard $S$, Dewailly $D$. Is the suppressive effect of cyproterone acetate on serum anti-Müllerian-hormone levels in women with polycystic ovary syndrome stronger than under oral contraceptive pill? Gynecol Endocrinol 2016;32:612-6.

22. Fábregues F, Castelo-Branco C, Carmona F, Guimerá M, Casamitjana R, Balasch J. The effect of different hormone therapies on anti-müllerian hormone serum levels in anovulatory women of reproductive age. Gynecol Endocrinol 2011;27:216-24.

23. Dursun F, Güven A, Yıldız M. Assessment of anti-Müllerian hormone level in management of adolescents with polycystic ovary syndrome. J Clin Res Pediatr Endocrinol 2016;8:55-60.

24. Panidis D, Georgopoulos NA, Piouka A, Katsikis I, Saltamavros $A D$, Decavalas $G$, et al. The impact of oral contraceptives and metformin on anti-Müllerian hormone serum levels in women with polycystic ovary syndrome and biochemical hyperandrogenemia. Gynecol Endocrinol 2011;27:587-92.

25. Somunkiran A, Yavuz T, Yucel O, Ozdemir I. Anti-Müllerian hormone levels during hormonal contraception in women with polycystic ovary syndrome. Eur J Obstet Gynecol Reprod Biol 2007;134:196-201.

26. Haisenleder DJ, Dalkin AC, Ortolano GA, Marshall JC, Shupnik MA. A pulsatile gonadotropin-releasing hormone stimulus is required to increase transcription of the gonadotropin subunit genes: evidence for differential regulation of transcription by pulse frequency in vivo. Endocrinology 1991;128:509-17.

27. Ehrmann DA. Polycystic ovary syndrome. N Engl J Med 2005;352:1223-36.

28. Chun S. Serum luteinizing hormone level and luteinizing hormone/follicle-stimulating hormone ratio but not serum anti-Müllerian hormone level is related to ovarian volume in Korean women with polycystic ovary syndrome. Clin Exp Reprod Med 2014;41:86-91.

29. Chun S. 1-h Postprandial glucose level is related to the serum anti-Müllerian hormone level in women with polycystic ovary syndrome. Gynecol Endocrinol 2015;31:815-8.

30. Abdul-Ghani MA, Lyssenko V, Tuomi T, DeFronzo RA, Groop L. Fasting versus postload plasma glucose concentration and the risk for future type 2 diabetes: results from the Botnia Study. Diabetes Care 2009;32:281-6.

31. Nardo LG, Yates AP, Roberts SA, Pemberton P, Laing I. The relationships between $\mathrm{AMH}$, androgens, insulin resistance and basal ovarian follicular status in non-obese 


\section{Obstetrics \& Gynecology Science}

Vol. 63, No. 1, 2020

subfertile women with and without polycystic ovary syndrome. Hum Reprod 2009;24:2917-23.

32. Skałba P, Cygal A, Madej P, Dąbkowska-Huć A, Sikora J, Martirosian $G$, et al. Is the plasma anti-Müllerian hormone $(\mathrm{AMH})$ level associated with body weight and metabolic, and hormonal disturbances in women with and without polycystic ovary syndrome? Eur J Obstet Gynecol Reprod Biol 2011;158:254-9.

33. Park $\mathrm{CH}$, Chun S. Association between serum gonadotropin level and insulin resistance-related parameters in Korean women with polycystic ovary syndrome. Obstet Gynecol Sci 2016;59:498-505.

34. Shim AR, Hwang IY, Lim KJ, Choi YM, Jeon YE, Seo SK, et al. Inappropriate gonadotropin secretion in polycystic ovary syndrome: the relationship with clinical, hormonal and metabolic characteristics. Korean J Obstet Gynecol 2011;54:659-65.

35. Caglar GS, Kahyaoglu I, Pabuccu R, Demirtas S, Seker R. Anti-Mullerian hormone and insulin resistance in classic phenotype lean PCOS. Arch Gynecol Obstet 2013;288:905-10.

36. Cassar S, Teede HJ, Moran LJ, Joham AE, Harrison CL, Strauss BJ, et al. Polycystic ovary syndrome and antiMüllerian hormone: role of insulin resistance, androgens, obesity and gonadotrophins. Clin Endocrinol (Oxf) 2014;81:899-906.

37. Monash University. International Evidence-based Guideline for the Assessment and Management of Polycystic Ovary Syndrome. Melbourne: Monash University; 2018. 